\title{
Gerechtigkeit als Desiderat der relationalen Rationalität
}

\author{
Walter Pfannkuche, Kassel
}

Die von ihm entwickelte modernisierte Version des Tianxia empfiehlt Zhao Tingyang als Modell zur Überwindung der mit der Globalisierung aufgetretenen oder verschärften Probleme globaler Konfrontation. Aus der modernen Perspektive sei die internationale Politik nur ein Anhängsel staatlicher Politik und bleibe dem Ziel verpflichtet, den Nutzen des einzelstaatlichen Akteurs zu maximieren. Die Politik der Nutzenmaximierung sei aber nicht in der Lage, Vertrauen und stabile Bündnisse hervorzubringen. Sie führe vielmehr zu immer raffinierteren Strategien der Steigerung des eigenen Nutzens, die aber bald von den Übervorteilten kopiert und für eine Revanche an den Ausbeutern genutzt würden. Die von solchen Dominierungsstrategien geprägte, feindselige Konkurrenz berge stets die Gefahr unkontrollierbarer Reaktion und führe die Welt an den Rand des Chaos.

Die Wurzel all dieser Übel entdeckt Zhao in einem Verständnis von Rationalität, demzufolge rationales Verhalten in der Maximierung des eigenen Nutzens besteht. Ausgehend von diesem Verständnis seien alle Versuche der Spieltheoretiker, den Konflikt der Egoismen in Kooperation zu überführen, gescheitert. Der einzige Ausweg bestehe darin, endlich zu erkennen, dass Koexistenz eine notwendige Bedingung der Bewahrung der eigenen Existenz ist. Das egozentrische Verständnis von Rationalität müsse daher durch ein relationales ersetzt werden. Diese Form der Rationalität räumt der gegenseitigen Schadensminimierung einen Vorrang vor der individuellen Nutzenmaximierung ein. Sie zielt auf die Schaffung einer stabilen, vertrauensbasierten Koexistenz, auf ein konfuzianisches Optimum, das alle Kooperierenden an Nutzenzuwächsen beteiligt.

Auch wenn man Zhaos Beschreibung der gegenwärtigen Probleme teilt, ist es zweifelhaft, dass seine Kritik am westlich-modernen Rationalitätsbegriff den Verursacher der Probleme korrekt identifiziert. Die relatio- 
nale Rationalität soll sich dadurch auszeichnen, dass sie Koexistenz als eine notwendige Bedingung der Bewahrung der eigenen Existenz anerkennt und so eine vertrauensvolle Kooperation ermöglicht. Aber selbst in spieltheoretischen Modellen konnte gezeigt werden, dass es auch auf der Basis des Eigeninteresses für alle Beteiligten am vorteilhaftesten ist, die psychische Disposition zu entwickeln, sich an Regeln zu orientieren, die nicht unmittelbar auf die Maximierung des Eigennutzens zielen. So realisieren die Beteiligten des von Mackie beschriebenen Soldaten-Dilemmas das für beide beste Resultat, wenn sie nicht versuchen, ihren individuellen Nutzen zu maximieren, sondern sich zuverlässig an Regeln der Solidarität in gefährlichen Situationen orientieren (Mackie 1981, 115-119). Und ganz analog hat Gauthier gezeigt, dass es rational ist, sich die Disposition eines „constrained maximizers“ anzueignen (Gauthier 1986, 177-184). Das bezeichnet die Disposition, anderen gegenüber stetig gewisse Rücksichten zu üben, um als vertrauenswürdig anerkannt und nicht von zukünftiger Kooperation ausgeschlossen zu werden. Wenn das überzeugende Resultate einer spieltheoretischen Rationalität sind, dann bleibt zu zeigen, inwiefern die relationale Rationalität darüber hinausgeht.

Ein gutes Argument für die Überlegenheit der relationalen Rationalität wäre es, wenn diese einen Gerechtigkeitsbegriff generieren könnte, der von allen Beteiligten geteilt werden und ein Handeln anleiten kann, das das Verlangen nach Revanche zum Verschwinden bringt. Gerade an dieser Stelle bleibt das Modell des Tianxia aber zu unbestimmt. Zhao verweist darauf, dass ein jeder Gerechtigkeitsbegriff sich durch die subjektiven Präferenzen der Menschen beweisen muss. Dieses subjektive Gerechtigkeitsempfinden sieht er durch drei Evidenzen charakterisiert: Evidenz 1 besagt, dass ein System nur dann legitim ist, wenn es auf der Zustimmung aller beruht. Da es diese aber nur selten geben wird, besagt Evidenz 2: Ein System ist nur dann legitim, wenn die Mehrheit ihm zustimmt. Ein auf Mehrheitsentscheidungen basierendes demokratisches System könne aber nicht garantieren, dass die getroffenen Entscheidungen den öffentlichen und individuellen Nutzen am besten steigern. Deshalb sei es erforderlich, auf eine dritte Evidenz zurückzugreifen: Ein System ist nur dann legitim, wenn es der ,Volksseele entspricht. Die Volksseele soll die Gesamtheit der Anschauungen repräsentieren, die sich als vorteilhaft erwiesen haben und von allen geteilt werden. Doch mit dieser dritten Evidenz wird das Modell zirkulär. Es verweist nun auf eine Einmütigkeit, von der im ersten Schritt zugestanden wurde, dass sie nur selten vorkommt. Und das wird insbesondere für Fragen der Gerechtig- 
keit gelten. Gerade hier gehen in modernen Gesellschaften die Meinungen weit auseinander. Ist es gerecht, wenn sich das Einkommen am ökonomischen Wert einer Leistung bemisst, oder handelt es sich dabei um eine ungerechtfertigte Kapitalisierung von Talent?

Erfolgversprechender scheint es deshalb, sich an Zhaos Überlegungen zum konfuzianischen Optimum zu orientieren. Dieses verlangt, dass alle an Nutzenzuwächsen beteiligt sein sollen. Das lässt natürlich einen weiten Spielraum für die Verteilung der durch die Kooperation generierten Nutzenzuwächse. Genügt es, wenn jeder wenigstens etwas profitiert, sollen alle gleichermaßen bessergestellt werden oder kommt es darauf an, dass die Position der am wenigsten Profitierenden so gut wie möglich ist? Leider liefert auch das konfuzianische Prinzip „Wenn du etwas erreichen willst, hilf anderen, etwas zu erreichen" keine Antwort. So könnte ein Kapitalist behaupten, er helfe anderen, ihre Ziele zu erreichen, indem er ihnen Arbeit und Einkommen bietet. Aber das macht eine kapitalistische Wirtschaftsordnung nicht gerecht. Zhao neigt dem Rawls'schen Differenzprinzip zu, möchte dieses aber gern auf eine andere Basis stellen. Er verweist dazu auf Laozi, der bereits erkannt habe, dass die übertriebene Steigerung des Nutzens Einzelner zu Gleichgewichtsstörungen und diese zur Katastrophe führen. Alles Bestehende könnte, so Laozi, aber nur weiterbestehen, wenn es sich im Gleichgewicht befinde, und deshalb sei eine Minderung des Nutzens der Starken auch im Interesse der Starken, weil dies auch deren Existenz sichere. Diese von Klugheit getragene Rücksicht der Starken auf die Schwachen führt aber nicht notwendig zur Anerkennung des Differenzprinzips. Stabilität kann es auch in Verteilungszuständen geben, die nicht dem Differenzprinzip genügen. Zudem verkehrt eine funktionale Definition der Gerechtigkeit, die als gerecht diejenigen Regelungen bezeichnet, die Stabilität gewährleisten, das Verhältnis zwischen den Begriffen: Eine Ordnung ist nicht deshalb gerecht, weil sie stabil ist, sondern sie ist deshalb stabil, weil sie von den Beteiligten für gerecht gehalten und damit innerlich akzeptiert wird.

Diese Unbestimmtheit lässt es notwendig erscheinen, das Konzept der relationalen Rationalität weiter zu präzisieren. Das System des Tianxia ist insofern bescheiden, als es nicht verspricht, die Glückseligkeit jedes Einzelnen herbeizuführen. Es soll vielmehr Garantien für Frieden und Sicherheit bereitstellen (Zhao 2020, 21). Aber Frieden und Sicherheit wird es jenseits repressiver Systeme nur geben können, wenn die Beteiligten ein Konzept der Gerechtigkeit teilen und auch erfahren können, dass dieses in ihrer Gemeinschaft realisiert wird. Für die Realisierung kann die Philoso- 
phie nicht aufkommen; die Formulierung normativ überzeugender Begriffe zur Überwindung der Probleme der globalen Moderne bleibt ihr weiterhin aufgegeben.

\section{Literatur}

Gauthier, David. 1986. Morals by Agreement, Oxford: Clarendon Press.

Mackie, John-Leslie. 1981. Ethics. Inventing Right and Wrong, New York: Penguin Books.

Zhao, Tingyang. 2020. Alles unter dem Himmel: Vergangenheit und Zukunft der Weltordnung, Berlin: Suhrkamp. 